PoS $\quad \begin{aligned} & \text { PROCEEDINGS } \\ & \text { OF SCIENCE }\end{aligned}$

\title{
Higgs boson measurements
}

\section{Roberto Salerno* on behalf of the ATLAS and CMS Collaborations}

Laboratoire Leprince-Ringuet, CNRS/IN2P3, École polytechnique

E-mail: roberto.salernodcern.ch

The latest results on Higgs boson physics obtained by ATLAS and CMS experiments using LHC proton-proton collisions at $\sqrt{s}=13 \mathrm{TeV}$ are presented.

European Physical Society Conference on High Energy Physics - EPS-HEP2019 -

10-17 July, 2019

Ghent, Belgium

${ }^{*}$ Speaker. 


\section{Introduction}

The discovery of the Higgs $(\mathrm{H})$ boson in 2012 by the ATLAS and CMS Collaborations [1, $2,3]$ has closed a chapter in particle physics and opened new avenues for research. Not only on the experimental side, putting an end to a decades-long search, but also, and more sharply, by completing the set of predictions by the Standard Model (SM). The landscape of Higgs boson physics has been extended extraordinarily since then. The LHC has delivered in Run2 (20152018) a dataset corresponding to an integrated luminosity close to $160 \mathrm{fb}^{-1}$ of $13 \mathrm{TeV}$ protonproton (pp) collisions, much larger than the $25 \mathrm{fb}^{-1}$ of collisions at 7 and $8 \mathrm{TeV}$ of Run1 (20112012). The ATLAS and CMS experiments $[4,5]$ have started to exploit this dataset, leading to substantial progress in the understanding of the Higgs boson properties: its couplings with all the third generation charged fermions have been established, as well as all the main Higgs boson production modes predicted by the SM. For some measurements, such as the total production cross section, the experimental uncertainties start to match those on the theory prediction. In parallel, no non-SM particles have been observed at the electroweak scale. This suggests a separation of thirdscales between the SM and the extra degrees of freedom of a more complete theory which would extend the SM. The new particles and interactions of the new theory may lie at energies much higher than the electroweak scale and would thus be difficult to directly probe at the LHC.

The Higgs boson landscape is currently divided in four main parts: the study of the Higgs boson profile; the move form discoveries to properties measurements; the search for rare decays and productions mechanisms; the search for Beyond SM (BSM) physics.

\section{The Higgs boson profile}

The Higgs boson mass $\left(\mathrm{m}_{H}\right)$ is the single parameter that is completely determined in the SM Higgs sector. To measure $\mathrm{m}_{H}$, the ATLAS and CMS Collaborations rely on the two high massresolution and sensitive channels, $H \rightarrow \gamma \gamma$ and $H \rightarrow Z Z \rightarrow 4 \ell$. The most precise measurements are obtained combing the individual decay channels and the LHC Run1 and Run2 datasets. The results are summarised in Table 1. The CMS measurement [7] has been released after EPS-HEP2019.

Table 1: A summary of the most precise measured Higgs boson mass from ATLAS and CMS Collaborations.

\begin{tabular}{c|c|c|c|c}
\hline \hline Exp. & Dataset & channels & $\mathrm{m}_{H} \pm$ tot. $( \pm$ stat. \pm sys. $)$ & reference \\
\hline ATLAS & Run1+Run2 $\left(36 \mathrm{fb}^{-1}\right)$ & $4 \ell+\gamma \gamma$ & $124.97 \pm 0.24( \pm 0.16 \pm 0.18) \mathrm{GeV}$ & {$[6]$} \\
CMS & Run1+Run2 $\left(36 \mathrm{fb}^{-1}\right)$ & $4 \ell+\gamma \gamma$ & $125.35 \pm 0.15( \pm 0.12 \pm 0.09) \mathrm{GeV}$ & {$[7]$} \\
\hline \hline
\end{tabular}

Another crucial parameter is the $\mathrm{H}$ boson total width $\left(\Gamma_{H}\right)$, important as well for BSM searches. The SM expectation of $\Gamma_{H}$ is around $4 \mathrm{MeV}$. The most precise method to measure the Higgs boson width is obtained from the relative measurements of the on-shell and off-shell production cross sections. Figure 1 shows the transverse mass $\mathrm{m}_{Z Z}^{T}$ distribution in the ATLAS analysis [8] used to extract the bound on the Higgs boson total width and the likelihood scans with the results from the CMS analysis [9]. The results are summarised in Table 1.

The precision on $\Gamma_{H}$ from the reconstructed mass lineshape is approximately $1 \mathrm{GeV}[10,11]$, which is significantly worse than the result from the off-shell method being limited by the detector 

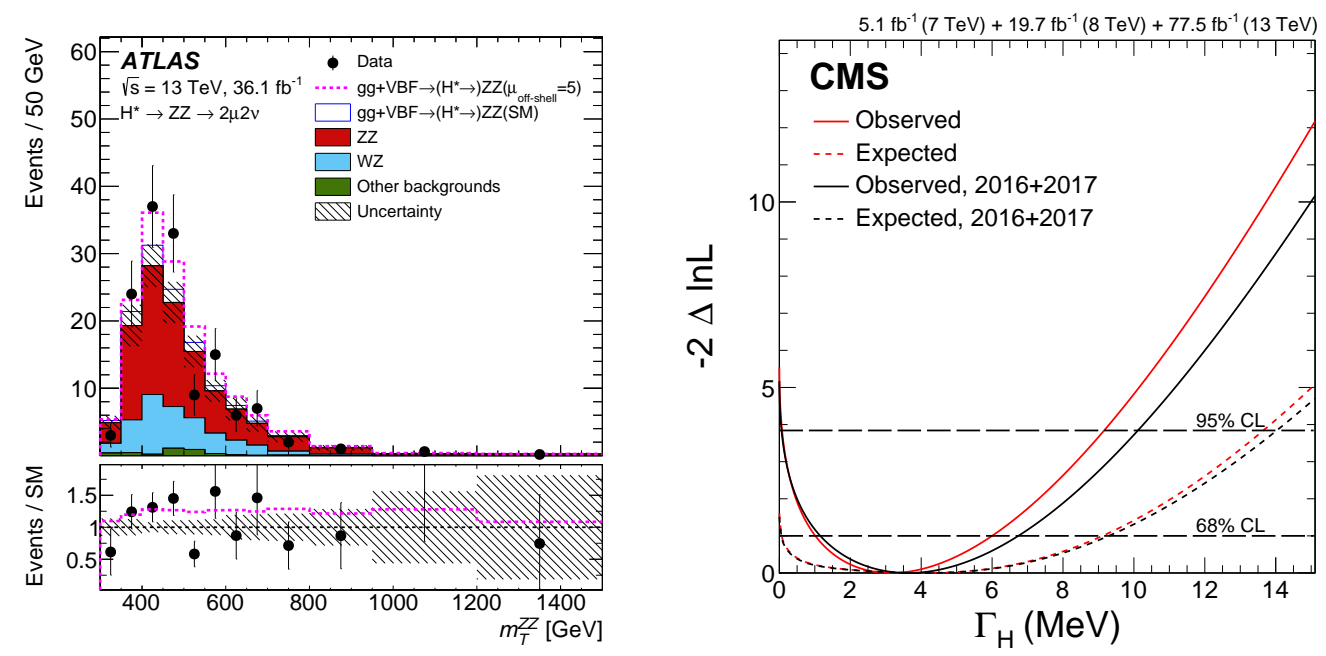

Figure 1: Observed transverse mass $\mathrm{m}_{Z Z}^{T}$ distribution in the $\mu \mu$ channel of the $\mathrm{ZZ} \rightarrow 2 \ell 2 v$ off-shell region, compared to the expected contributions from the SM including the Higgs boson (stacked) from ATLAS analysis [8] (left). Observed (solid) and expected (dashed) likelihood scans of $\Gamma_{H}$ from CMS analysis [9] (right).

Table 2: A summary of the results from ATLAS and CMS Collaborations using off-shell method to measure the Higgs boson total width.

\begin{tabular}{c|c|c|c|c}
\hline \hline Exp. & Dataset & channels & obs. 95\% C.L. on $\Gamma_{H}$ & reference \\
\hline ATLAS & Run2 $\left(36 \mathrm{fb}^{-1}\right)$ & $4 \ell+2 \ell 2 v$ & $\Gamma_{H}<14.4 \mathrm{MeV}$ & {$[8]$} \\
CMS & Run1+Run2 $\left(77.5 \mathrm{fb}^{-1}\right)$ & $4 \ell$ & $0.08<\Gamma_{H}<9.6 \mathrm{MeV}$ & {$[9]$} \\
\hline \hline
\end{tabular}

resolution. The constraint on the $\mathrm{H}$ boson lifetime is equivalent to a lower bound on $\mathrm{t} \Gamma_{H}$ and it was derived from the flight distance in the CMS detector as $\Gamma_{H}>3.5 \times 10^{-9} \mathrm{MeV}$ at $95 \%$ CL [12].

All the Higgs boson couplings are fixed unambiguously once all the particle masses are known. Any deviation in the measurement of the couplings could therefore signal physics beyond the SM. The $\mathrm{H} \rightarrow \mathrm{ZZ} \rightarrow 4 \ell$ final state was updated by both ATLAS and CMS Collaborations using the whole LHC Run2 dataset. More that 200 candidates of $\mathrm{H}$ boson are expected for each experiment after the selections in this final state. Figure 2 show the four-lepton invariant mass distributions for the selected Higgs boson candidates in the ATLAS [13] and CMS [14] analyses.

Furthermore, the Higgs boson candidate events are classified into several categories providing sensitivity to different production modes in various regions of phase space. To perform the categorisations both full event topology and MVA/ME discriminants exploiting full decay and production information are used. The production cross sections times branching fraction for $\mathrm{H} \rightarrow \mathrm{ZZ}$ decay measured in dedicated production bins and normalised by the SM expectation are shown in Figure 3. The measurements are performed within the Simplified Template Cross Sections (STXS) framework [15] using the Stage-0 bins, where just the H boson production mechanisms are probed, and the reduced Stage-1.1 bins, where a finer split of kinematic regions is performed. The measurements are in agreement with the SM predictions.

The combined measurement of the total cross section of $\mathrm{H}$ boson production is performed [16, 

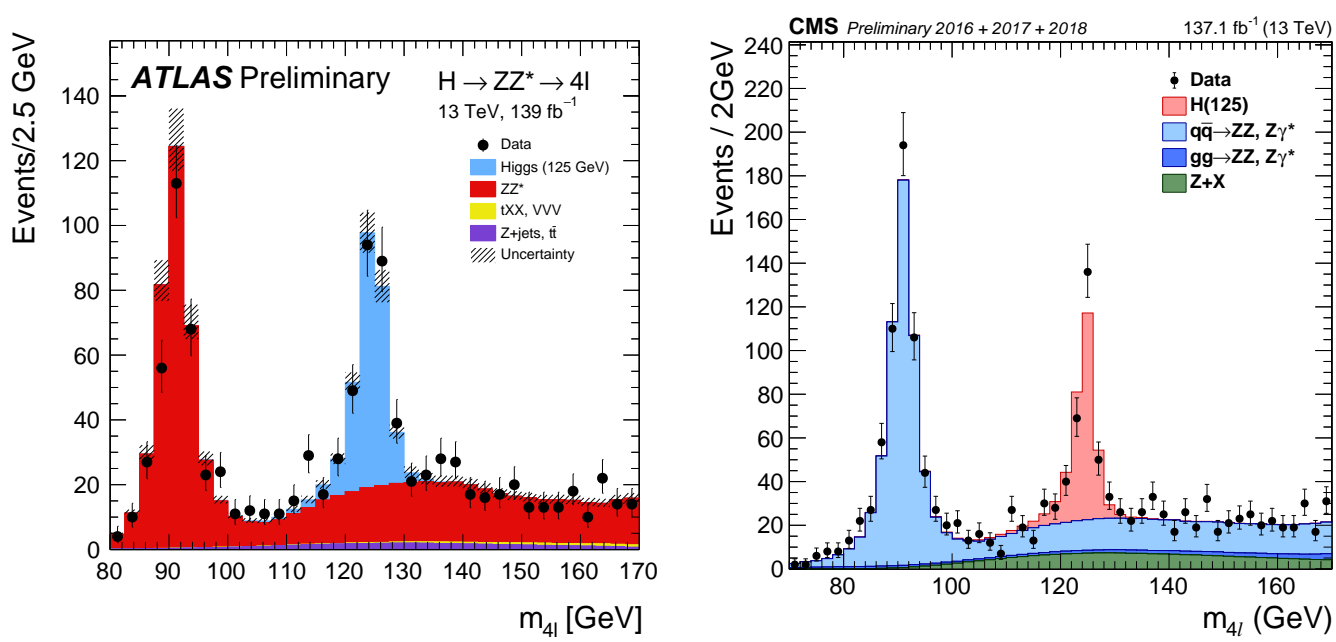

Figure 2: Four-lepton invariant mass distributions for the selected Higgs boson candidates in the ATLAS [13] (right) and CMS [14] (left) analyses.
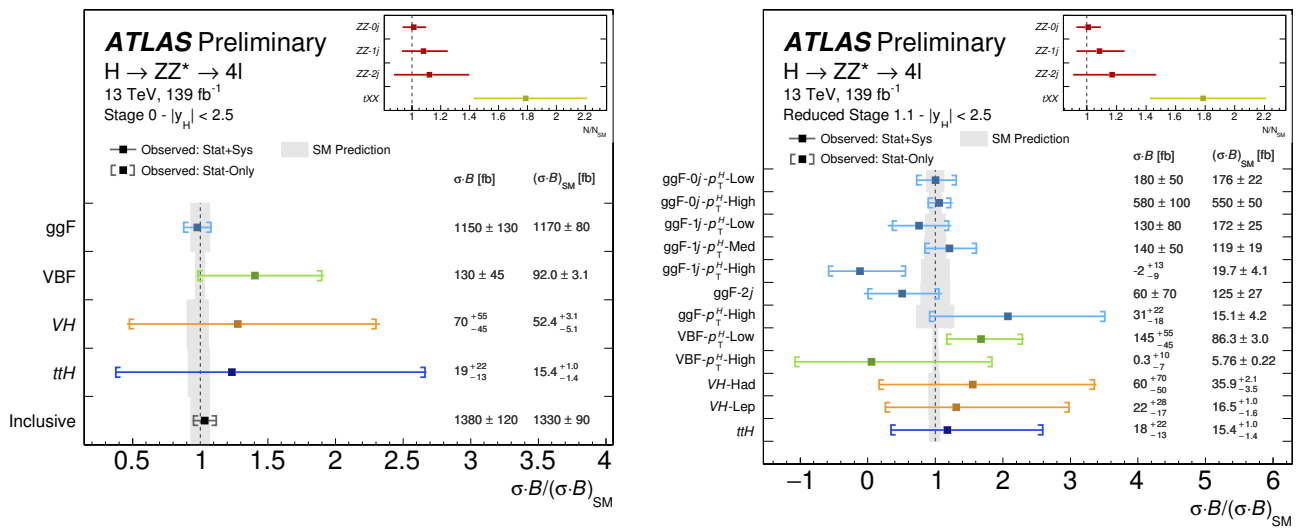

Figure 3: The observed and expected SM values of the cross section ratios normalised by the SM expectation for (left) the inclusive production and in the Stage- 0 and (right) the reduced Stage-1.1 production bins defined in the STXS framework. The fitted normalisation factors for the the ZZ and tXX background are shown in the inserts. Different colours indicate different Higgs boson production modes (or background sources). The grey vertical band represents the theory uncertainty in the signal prediction. Results are from [13].

17] from the measured $\mathrm{H} \rightarrow \mathrm{ZZ} \rightarrow 4 \ell$ and $\mathrm{H} \rightarrow \gamma \gamma$ event yields, which are combined accounting for luminosity, detector effects, acceptances, and branching fractions. Table 3 summarises measurements of the total cross section of $\mathrm{H}$ boson production in $\mathrm{H} \rightarrow \mathrm{ZZ} \rightarrow 4 \ell$ and $\mathrm{H} \rightarrow \gamma \gamma$ decay channels and their combination. The measurements are in agreement with the SM prediction.

Different cross section measurements are also performed in the $\mathrm{H} \rightarrow \gamma \gamma$ and $\mathrm{H} \rightarrow \mathrm{ZZ} \rightarrow 4 \ell$ channels $[13,14,18]$, using the full LHC Run2 dataset, in various specific observables $\left(\mathrm{p}_{T}^{\gamma \gamma}, \mathrm{p}_{T}^{4 \ell},\left|y_{\gamma \gamma}\right|\right.$, $\left.\left|y_{4 \ell}\right|, \mathrm{N}_{j e t s}, \mathrm{p}_{T}^{j e t} 1, \mathrm{~m}_{j j}, \ldots\right)$ and they are compared with different predictions. Figure 5 shows a few examples of such differential cross sections measurements.

Using four differential cross sections measurements performed in the $\mathrm{H} \rightarrow \gamma \gamma$ analysis $\left(\mathrm{p}_{T}^{\gamma \gamma}\right.$, $\left|y_{\gamma \gamma}\right|, \mathrm{m}_{j j}, \delta \phi_{j j}$, and $\left.\mathrm{p}_{T}^{j e t 1}\right)$, constraints on the Wilson coefficients of both the SILH basis of the 
Table 3: A summary of the measurements of the total cross section of $\mathrm{H}$ boson production done by the ATLAS and CMS Collaborations in $\mathrm{H} \rightarrow \mathrm{ZZ} \rightarrow 4 \ell$ and $\mathrm{H} \rightarrow \gamma \gamma$ decay channels and their combination.

\begin{tabular}{|c|c|c|c|c|}
\hline Exp. & Dataset & Channels & Total production cross section & Reference \\
\hline \multirow{3}{*}{ ATLAS } & $\operatorname{Run} 2\left(139 \mathrm{fb}^{-1}\right)$ & $\gamma \gamma$ & $56.7_{-6.2}^{+6.4} \mathrm{pb}$ & \multirow{3}{*}{ [16] } \\
\hline & $\operatorname{Run} 2\left(139 \mathrm{fb}^{-1}\right)$ & $4 \ell$ & $54.4_{-5.4}^{+5.6} \mathrm{pb}$ & \\
\hline & $\operatorname{Run} 2\left(139 \mathrm{fb}^{-1}\right)$ & $4 \ell+\gamma \gamma$ & $55.4_{-3.1}^{+3.1}$ (stat) ${ }_{-2.8}^{+3.0}($ syst $) \mathrm{pb}$ & \\
\hline \multirow{3}{*}{ CMS } & $\operatorname{Run} 2\left(36 \mathrm{fb}^{-1}\right)$ & $\gamma \gamma$ & $64.4_{-9.6}^{+9.6} \mathrm{pb}$ & \multirow{3}{*}[17]{} \\
\hline & $\operatorname{Run} 2\left(36 \mathrm{fb}^{-1}\right)$ & $4 \ell$ & $58.2_{-9.8}^{+9.8} \mathrm{pb}$ & \\
\hline & $\operatorname{Run} 2\left(36 \mathrm{fb}^{-1}\right)$ & $4 \ell+\gamma \gamma$ & $61.1_{-6.0}^{+6.0}(\text { stat })_{-3.7}^{+3.7}($ syst $) \mathrm{pb}$ & \\
\hline \multicolumn{3}{|c|}{ SM prediction } & $55.6 \pm 2.5 \mathrm{pb}$ & [15] \\
\hline
\end{tabular}

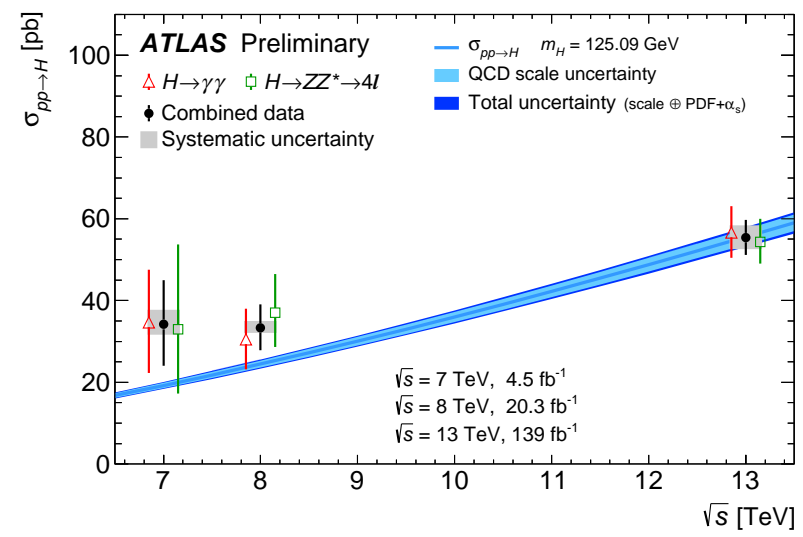

Figure 4: Total $\mathrm{pp} \rightarrow \mathrm{H}+\mathrm{X}$ cross sections measured at center-of-mass energies of 7,8 , and $13 \mathrm{TeV}$, compared to $\mathrm{SM}$ predictions. The $\mathrm{H} \rightarrow \gamma \gamma$ channel (red triangles), $\mathrm{H} \rightarrow \mathrm{ZZ} \rightarrow 4 \ell$ channel (green squares) and combined (black dots) measurements are shown. The grey bands on the combined measurements represent the systematic uncertainty, while the error bars show the total uncertainty. The light blue band shows the estimated uncertainty due to missing higher-order corrections, and the dark blue band indicates the total uncertainty. Results are from [16].

Higgs Effective Lagrangian [19] (referred to as SILH) and the Warsaw basis of the SMEFT Lagrangian [20, 21] (referred to as SMEFT) have been set [18]. Figure 6 shows the ratios of the expected differential cross sections to the SM predictions for some representative values of the Wilson coefficients of the SMEFT CP-even operators and the limits on SMEFT CP-even and CPodd Wilson coefficients.

\section{From discoveries to property measurements}

The first observation of the $\mathrm{H}$ boson in the $\tau \tau$ final state was reported independently by the ATLAS [22] and CMS [23] experiments. In the recent analysis the CMS experiment has double the integrated luminosity analysed [24] and the cross section measurements in the $\mathrm{H} \rightarrow \tau \tau$ deacy channel are started to be performed split by production modes and in different kinematic regimes following the recommendation of the STXS framework. Some improvements have been introduced with respect to the previous analysis, namely the usage of a machine learning technique for cate- 

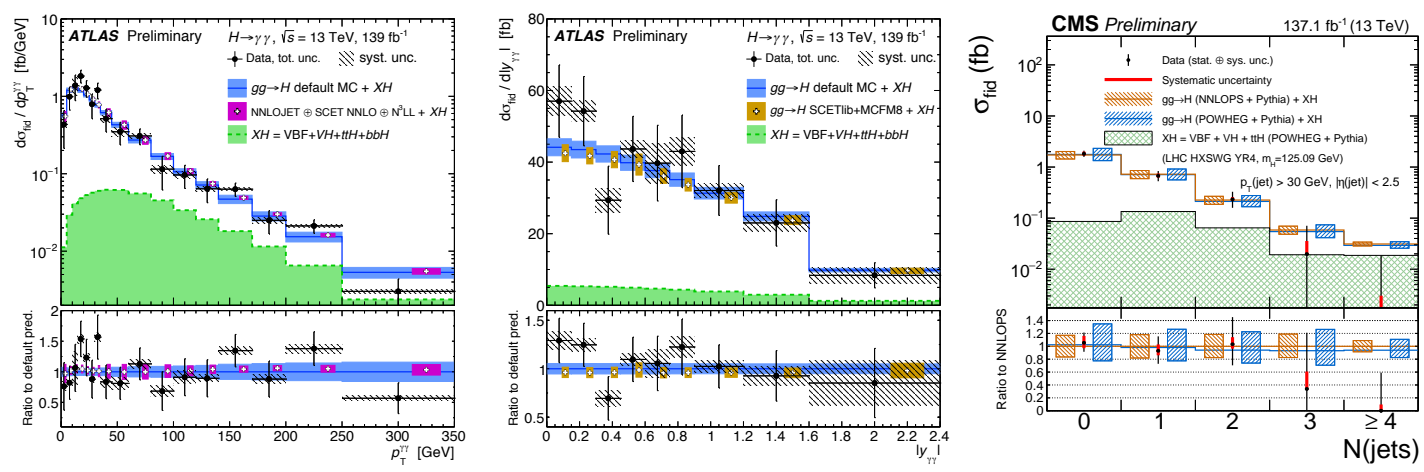

Figure 5: The differential cross sections measurements as a function of the diphoton kinematics in the $\mathrm{H} \rightarrow \gamma \gamma$ channel for $\mathrm{p}_{T}^{\gamma \gamma}$ (left) and $\left|y_{\gamma \gamma}\right|$ (center), figures are taken from [18]. The differential cross section measurement as a function of the number of jets, $\mathrm{N}_{\text {jets }}$, in the $\mathrm{H} \rightarrow \mathrm{ZZ} \rightarrow 4 \ell$ channel (right), figure is taken from [14].
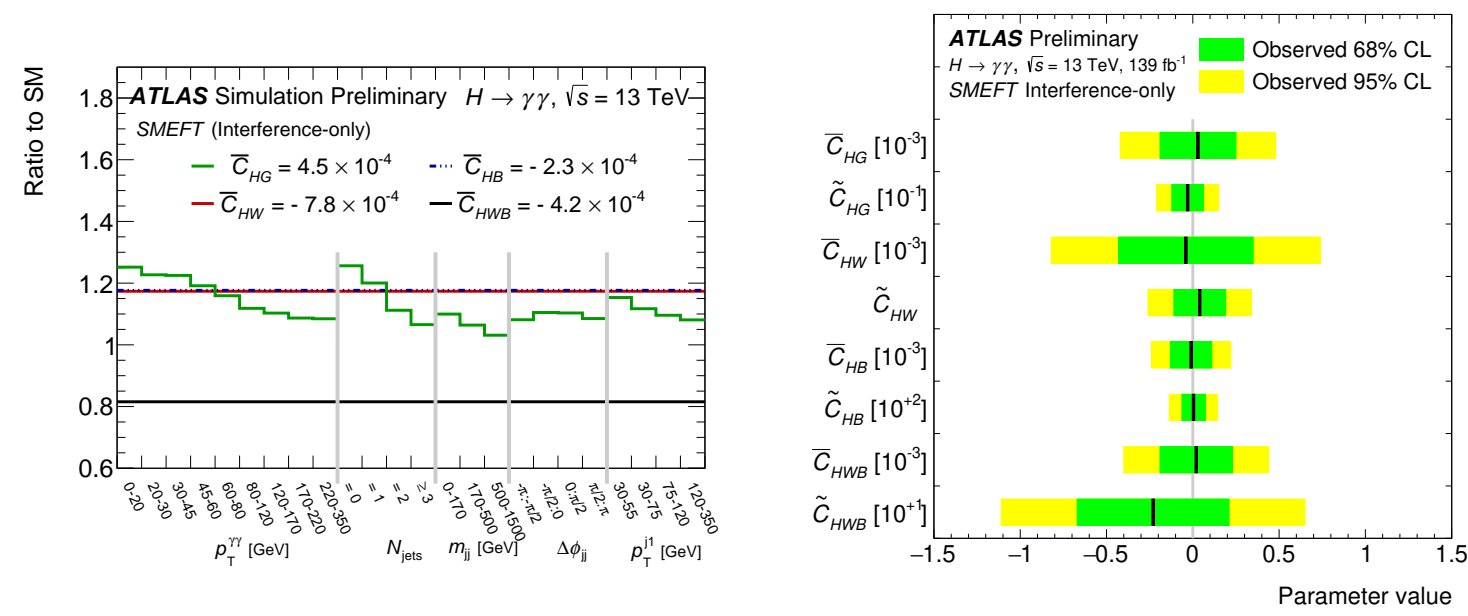

Figure 6: The effect on the five differential distributions used in the analysis of the $\mathrm{CP}$-even coefficients $C_{H G}$, $C_{H B}, C_{H W}$, and $C_{H W B}$ of the SMEFT effective Lagrangian for values of the coefficients close to the observed limits (left). Observed 68\% and 95\% CL limits on SMEFT CP-even and CP-odd Wilson coefficients. Limits are derived fitting one Wilson coefficient at a time while setting the other coefficients to zero (right). Results are from [18].

gorisation and the estimation of $90 \%$ of backgrounds using data-driven methods. Figure 7 shows the results of the two-dimensional fit for the signal strength of the $g g \rightarrow \mathrm{H}+\mathrm{bbH}$ and $\mathrm{VBF}+\mathrm{V}(\mathrm{qq}) \mathrm{H}$ production modes and the production cross sections times branching fraction for $\mathrm{H} \rightarrow \tau \tau$ decay channel measured in dedicated production bins and normalised by the SM expectation.

Another Yukawa coupling with third generation fermion recently observed by the ATLAS [25] and CMS [26] experiments is the top quark-H boson coupling. This coupling is only directly accessible when the $\mathrm{H}$ boson is produced in association with 1 or $>1$ top quarks, namely in the $\mathrm{ttH}$, tHq, and tHW production mechanisms. The observations were obtained from the combination of different final states: $\mathrm{ttH}(\mathrm{bb}), \mathrm{ttH}(\mathrm{WW}), \operatorname{ttH}(\tau \tau), \mathrm{ttH}(\mathrm{ZZ})$, and $\mathrm{ttH}(\gamma \gamma)$. It should be noted that the highest yield final state, $\mathrm{ttH}(\mathrm{bb})$, is systematics limited and has a low signal over background ratio, 

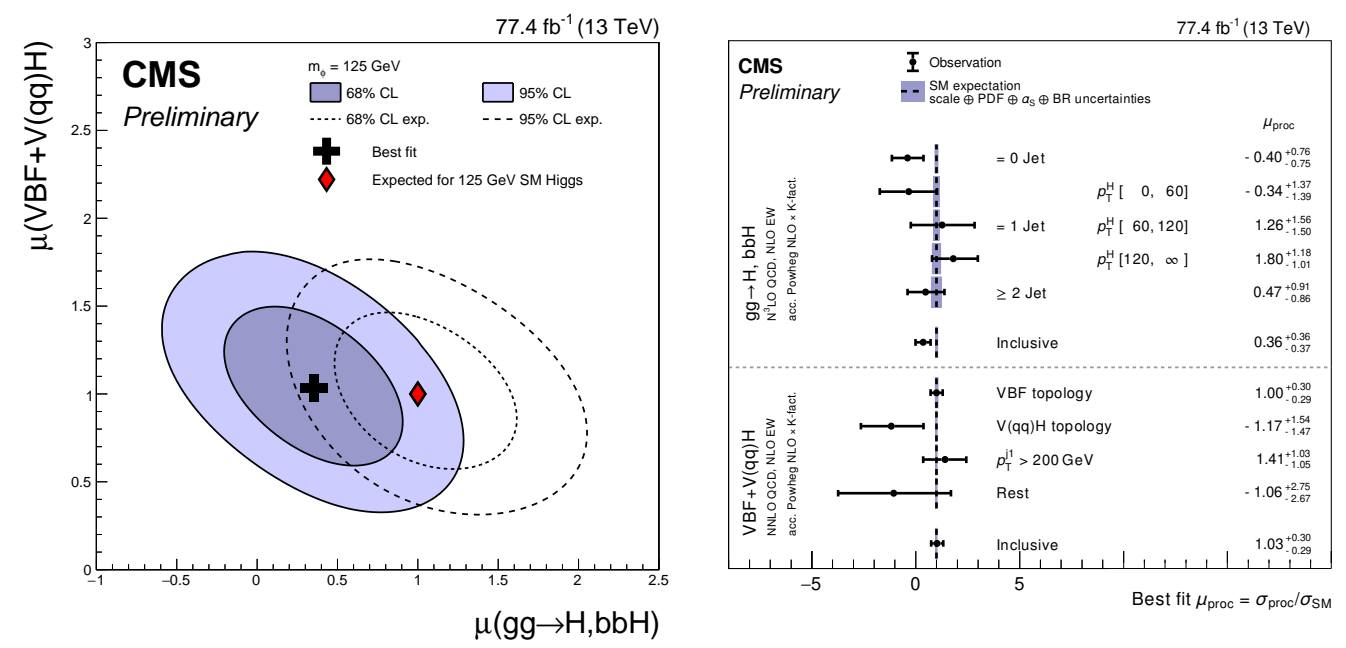

Figure 7: Two-dimensional contours corresponding to $68 \%$ and $95 \%$ confidence level (CL) for the signal strength of the $g g \rightarrow \mathrm{H}, \mathrm{bbH}$ and $\mathrm{VBF}+\mathrm{V}$ (qq)H production modes (left). The observed and expected SM values of the cross section ratios normalised by the SM expectation for the inclusive production and in production bins defined in the STXS framework (right). The purple vertical bands represent the theory uncertainty in the signal prediction. Results are from [24].

while the lowest yield final states, $\mathrm{ttH}(\mathrm{ZZ})$ and $\mathrm{ttH}(\gamma \gamma)$, have an excellent signal over background ratio and are statistically limited. With respect to the observations analyses more data, up to the full LHC Run2 dataset, has been added in few analyses [13, 14, 27, 28, 29, 30] moving towards the direct observation in most of the final state. Examples of the final discriminant variables for the $\mathrm{ttH}(\mathrm{bb}) \mathrm{CMS}$ analysis [27] and $\mathrm{ttH}(\gamma \gamma)$ ATLAS analysis [30] are shown in Figure 8. Table 4 summarises the measured signal strengths in each final states and the LHC Run2 analysed integrated luminosity.

Table 4: A summary of the measured signal strength in each ttH final states and the LHC Run2 analysed integrated luminosity. Analyses with ${ }^{\dagger}$ have been updated, including more luminosity with respect the observation papers $[25,26]$.

\begin{tabular}{c|c|c|c|c|c}
\hline \hline Exp. & & $\mathrm{ttH}(\mathrm{bb})$ & $\mathrm{ttH}(\mathrm{WW} / \tau \tau)$ & $\mathrm{ttH}(\gamma \gamma)$ & $\mathrm{ttH}(\mathrm{ZZ})$ \\
\hline \multirow{2}{*}{ ATLAS } & Run2 luminosity & $36 \mathrm{fb}^{-1}$ & $36 \mathrm{fb}^{-1}$ & $139 \mathrm{fb}^{-1} \dagger$ & $139 \mathrm{fb}^{-1}$ \\
& obs. signal strength & $0.79_{-0.60}^{+0.61}$ & $1.56_{-0.40}^{+0.42}$ & $1.38_{-0.36}^{+0.41}$ & $1.23_{-0.86}^{+1.44}$ \\
\hline \multirow{2}{*}{$\mathrm{CMS}$} & Run2 luminosity & $77 \mathrm{fb}^{-1} \dagger$ & $77 \mathrm{fb}^{-1} \dagger$ & $77 \mathrm{fb}^{-1} \dagger$ & $139 \mathrm{fb}^{-1} \dagger$ \\
& obs. signal strength & $1.15_{-0.29}^{+0.32}$ & $0.96_{-0.31}^{+0.34}$ & $1.7_{-0.5}^{+0.6}$ & $0.13_{-0.13}^{+0.92}$ \\
\hline \hline
\end{tabular}

The last Yukawa coupling with third generation fermion, the bottom quark-H boson coupling, is study in the $\mathrm{H} \rightarrow \mathrm{bb}$ decay channel. This is a difficult channel despite the large $\mathrm{BR}(\mathrm{H} \rightarrow \mathrm{bb})=58 \%$ due to large background. The coupling has been established [31,32] with a significance greater than 5 standard deviations combining all the $\mathrm{H}$ boson production mechanisms, in particular $\mathrm{VH}$ is the most sensitive one but ggF, VBF, and ttH play a role. In addition, the ATLAS Collaboration measured the cross-sections $\mathrm{VH}(\mathrm{bb})$ as a function of the gauge boson transverse momentum and interpreted the measurements to set limits on the parameters of an effective Lagrangian sensitive to 

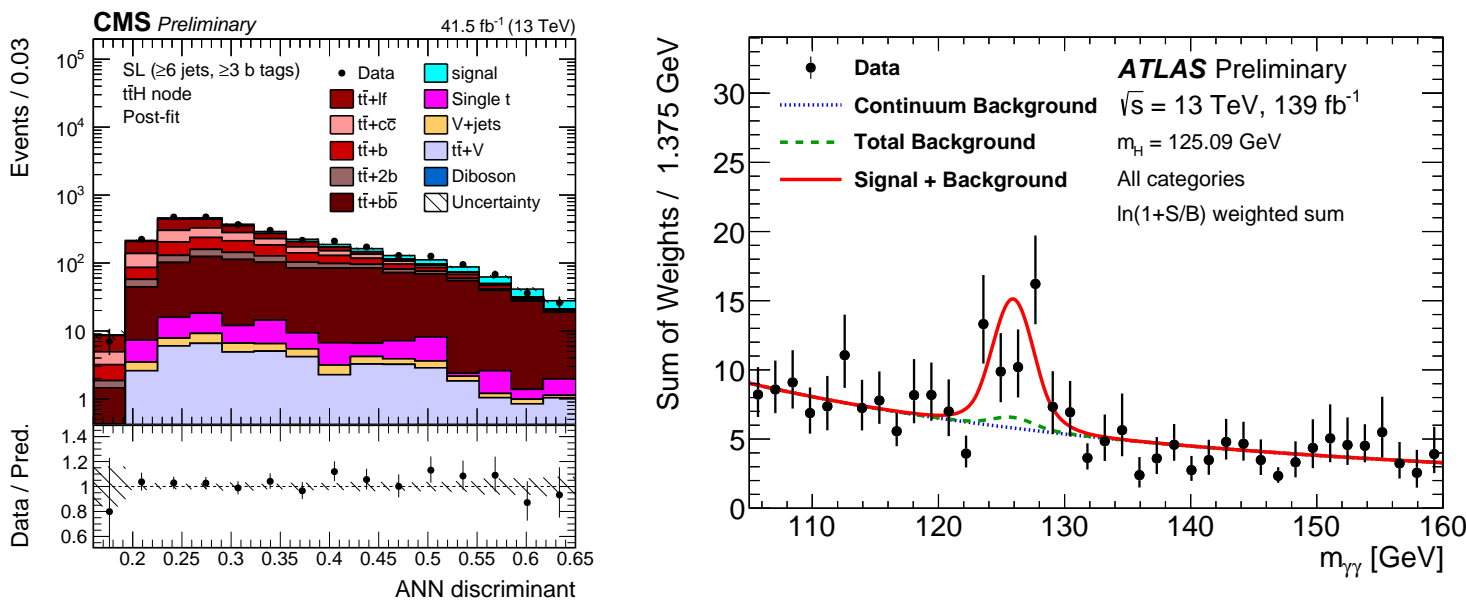

Figure 8: The discriminant shape of the ANN discriminant of the highest sensitivity category in dilepton channels for the $\mathrm{ttH}(\mathrm{bb}) \mathrm{CMS}$ analysis [27] after the fit on data (left). The expected background and signal contributions are stacked. The hatched uncertainty bands include the total uncertainty of the fit model. The distributions observed in data (markers) are overlayed. The first and the last bins include underflow and overflow events, respectively. The lower plots show the ratio of the data to the background prediction. The weighted diphoton invariant mass spectrum for the sum of all BDT categories observed in the $\mathrm{ttH}(\gamma \gamma)$ ATLAS analysis [30] (right). The error bars represent 68\% confidence intervals of the weighted sums. The solid red curve shows the fitted signal-plus-background model with the Higgs boson mass constrained to $125.09 \pm 0.24 \mathrm{GeV}$. The non-resonant and total background components of the fit are shown with the dotted blue curve and dashed green curve.

modifications of the Higgs boson couplings to the electroweak gauge bosons [33], a few results are summarised in Figure 9.
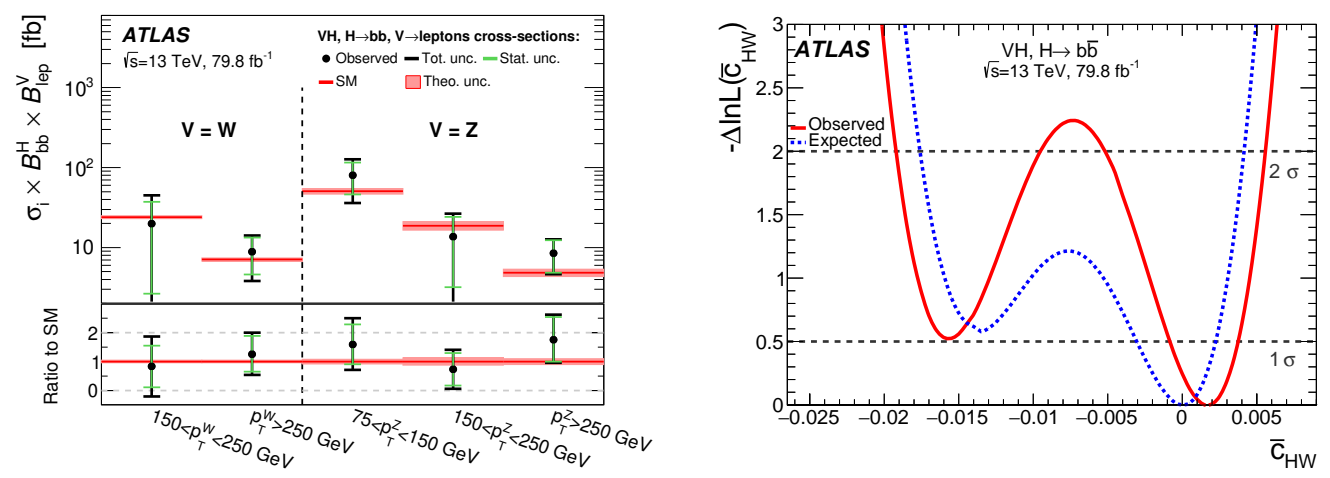

Figure 9: Measured VH, V $\rightarrow$ leptons reduced stage-1 STXS times the $\mathrm{H} \rightarrow$ bb branching fraction (left). The observed (solid) and expected (dotted) profiled negative-log-likelihood functions for the one-dimensional fits to constrain the coefficients $\mathrm{C}_{H W}$ of the SMEFT effective Lagrangian. Results are from [33].

\section{Rare decay and production mechanisms}

The $\mathrm{H} \rightarrow \mu \mu$ final state is used to probe Yukawa coupling with second generation fermion, namely the $\mu-\mathrm{H}$ boson coupling. The $\mu$ are the easiest objects to identify and measure, but small 
$\mathrm{B}(\mathrm{H} \rightarrow \mu \mu)=2 \times 10^{-4}$ (that means $\left.\mathscr{O}(5-6) \mathrm{evt} / \mathrm{fb}^{-1}\right)$, large backgrounds $\left(Z / \gamma^{*}\right.$, Diboson, Top), and the small signal over background regime make $\mathrm{H} \rightarrow \mu \mu$ a very challenging analysis. Critical are an excellent muon momentum resolution and sophisticated techniques to categorise and select events. The ATLAS Collaboration performed a recent analysis based on full LHC Run2 data [34], the main introduced improvements are a BDT-based event classification, a background modelling, and a better rejection of pile-up jet. Figure 10 shows the final dimuon invariant mass spectra after the selections for the ATLAS [34] and CMS [35] analyses. Table 5 summarise the observed and expected results.
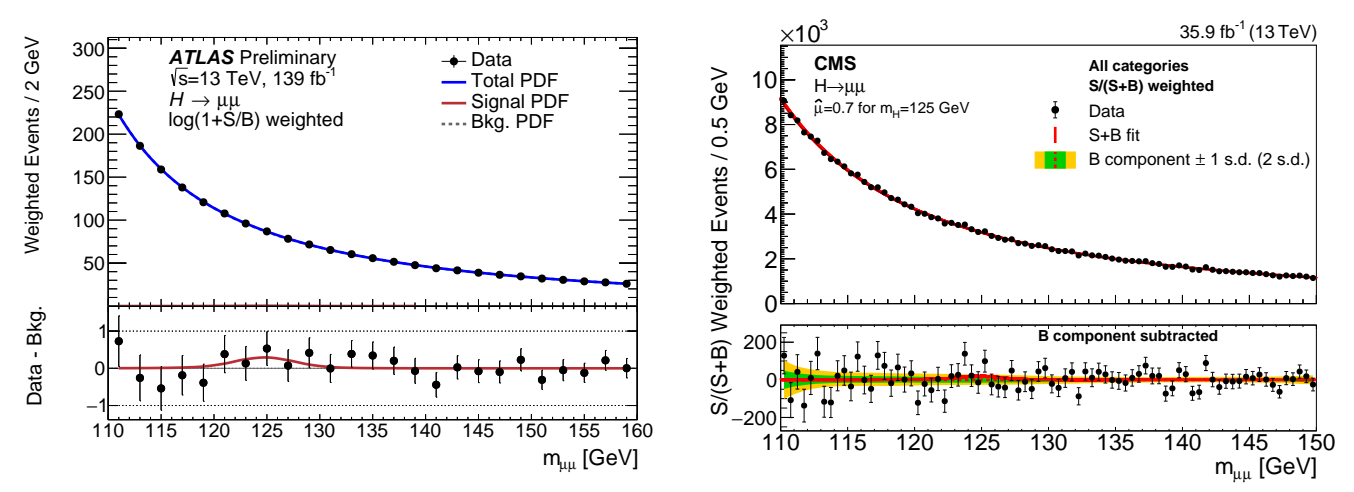

Figure 10: Data and weighted sum of signal-plus-background fits to each category. Events are weighted according to the expected signal-to-background ratio in the category to which they belong. Results are from the ATLAS analysis [34] (left) and CMS analysis [35] (right).

Table 5: Observed and expected results of the ATLAS and CMS H $\rightarrow \mu \mu$ analyses [34, 35].

\begin{tabular}{c|c|c|c|c}
\hline \hline Exp. & Dataset & obs(exp) UL on $\sigma / \sigma_{S M}$ & obs(exp) $\mu$ & obs(exp) sign. \\
\hline ATLAS & Run2 $\left(139 \mathrm{fb}^{-1}\right)$ & $1.7(1.3)$ & $0.5 \pm 0.7(1.0 \pm 0.7)$ & $0.8 \sigma(1.5 \sigma)$ \\
\hline CMS & Run1+Run2 $\left(36 \mathrm{fb}^{-1}\right)$ & $2.9(2.2)$ & $1.0 \pm 1.0(1.0 \pm 1.0)$ & $0.9 \sigma(1.0 \sigma)$ \\
\hline \hline
\end{tabular}

The charm quark-H boson coupling, another Yukawa coupling with second generation fermions, even if it has the same strength of the $\tau$-H boson coupling is way harder to probe. The reasons are that $\mathrm{BR}(\mathrm{H} \rightarrow \mathrm{cc})$ is only $0.05 \times \mathrm{B}(\mathrm{H} \rightarrow \mathrm{bb}), \mathrm{H} \rightarrow \mathrm{bb}$ is background for the analysis, there is a large (hadronic) background, and the charm jet ID is highly challenging. To probe this coupling complementary approaches exist : the direct search for $\mathrm{H} \rightarrow \mathrm{cc}$ decay, extract constraints on the coupling from kinematics, searches for charmonium decays, and the total width/global analysis. The CMS Collaboration has recently released a $\mathrm{H} \rightarrow \mathrm{cc}$ analysis [36] targeting the $\mathrm{VH}$ production mechanism and using $36 \mathrm{fb}^{-1}$ of LHC Run2 data. The CMS search uses all the three exclusive channels to capture $\mathrm{V}$ decay modes ( 0 . 1 , and 2 leptons), two approaches to explore the $\mathrm{H} \rightarrow \mathrm{cc}$ decay topology (resolved and merged), and advanced charm-tagging techniques are exploited. The observed (expected) upper limit on the production cross section times the decay branching ration divided by the SM expectation is $70\left(37_{-10}^{+16}\right)$ at $95 \% \mathrm{CL}$. The corresponding ATLAS analysis is described here [37] and its observed (expected) upper limit on the production cross section times the decay branching fraction divided by the SM expectation is $150(110)$ at $95 \%$ CL.

Finally, another important rare production mechanism is the Higgs boson pair $(\mathrm{HH})$ production. Due to this process we can access the Higgs boson trilinear coupling whose measurement will 
provide an independent test of the SM and verification that the Higgs mechanism is truly responsible for the EWSB by giving access to the shape of the Higgs scalar field potential. Both the ATLAS and CMS Collaborations performed studies of Higgs boson pair production and the most sensitive results are obtained combined different final states [38, 39]. The ATLAS analysis set an observed (expected) upper limit on the $\mathrm{HH}$ production cross section times the standard model value at $95 \%$ CL of 6.9 (10) while the corresponding CMS analysis of 22.2 (12.8).

\section{BSM Higgs boson sector}

There is a broad range of searches in the context of BSM physics within the ATLAS and CMS Collaborations. Different avenues are exploited: direct searches for additional neutral or charged Higgs bosons and exotic decays of the observed $\mathrm{H}$ boson. For all of them no significant deviations have been observed in the data with respect to the SM predictions, upper limits have been set and interpreted in specific models. As an example Figure 11 shows the observed and expected $95 \%$ CL upper limits for $\mathrm{m}_{A}$ versus the MSSM parameter $\tan \beta$ in the $M_{h}^{125}$ and hMSSM benchmark scenarios including in the same plot different Run2 CMS analyses.
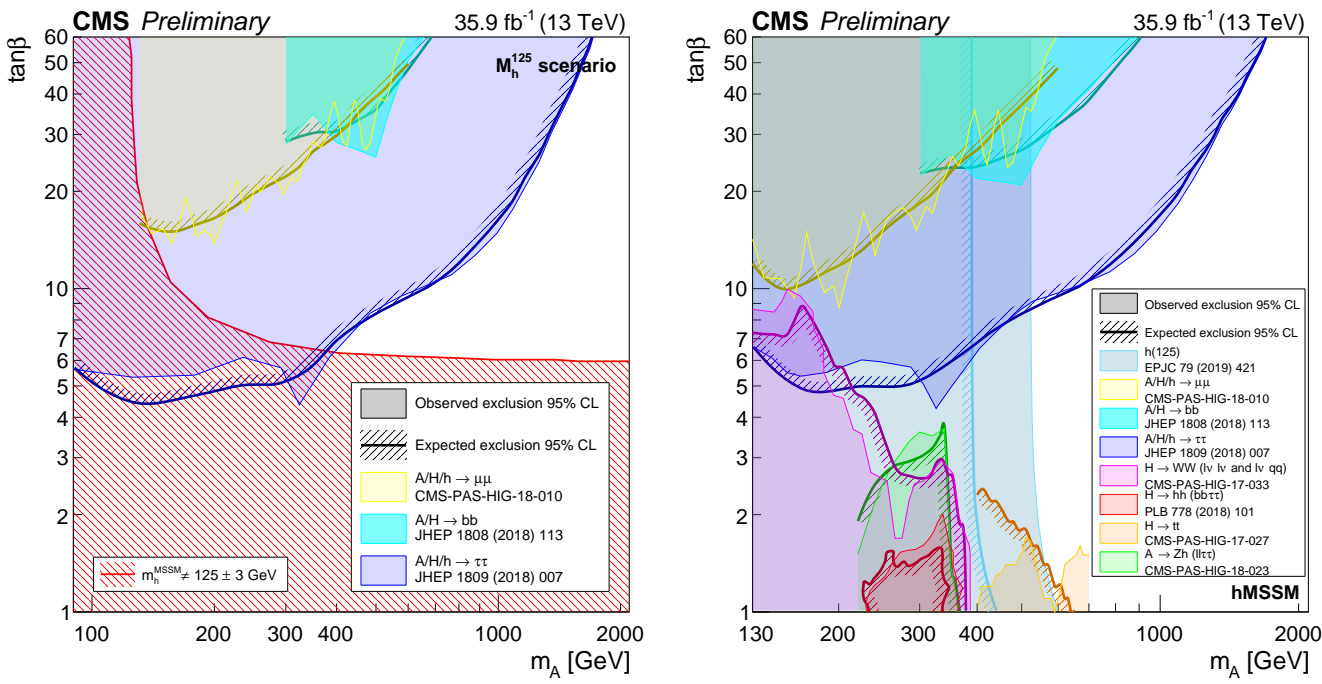

Figure 11: Observed and expected 95\% CL upper limits for $\mathrm{m}_{A}$ versus the MSSM parameter $\tan \beta$ in the $M_{h}^{125}$ scenario and in the hMSSM benchmark scenario (right).

\section{Conclusions}

A broad Higgs physics program is ongoing within ATLAS and CMS using the LHC Run2 dataset, which corresponds of less than 5\% of the final HL-LHC integrated luminosity. The landscape of Higgs boson physics is evolving on four main axes. First of all, the start of the precision era in the Higgs boson gauge sector, we are moving towards less than $10 \%$ uncertainties in the measurements. Then, the switch from discovery to properties measurements using the third-generation couplings. After that, the focus on rare processes, in particular towards the evidence/observation of second-generation coupling using LHC data and the probe of charm- $\mathrm{H}$ interaction and Higgs self-coupling towards HL-LHC. Finally, an extensive program of BSM searches is on-going. 


\section{References}

[1] ATLAS Collaboration, Phys. Lett. B 716 (2012) 1

[2] CMS Collaboration, Phys. Lett. B 716 (2012) 30

[3] CMS Collaboration, JHEP 06 (2013) 081

[4] ATLAS Collaboration, JINST 3 S08003 (2008)

[5] CMS Collaboration, JINST 3 S08004 (2008)

[6] ATLAS Collaboration, Phys. Lett. B784 (2018) 345

[7] CMS Collaboration, CMS-PAS-HIG-19-004, https://cds.cern.ch/record/2691211

[8] ATLAS Collaboration, Phys. Lett. B 786 (2018) 223

[9] CMS Collaboration, Phys. Rev. D 99 (2019) 112003

[10] ATLAS Collaboration, Phys. Rev. D 90 (2014) 052004

[11] CMS Collaboration, JHEP 11 (2017) 047

[12] CMS Collaboration, Phys. Rev. D 92 (2015) 072010

[13] ATLAS Collaboration, ATLAS-CONF-2019-025, https://cds.cern.ch/record/2682107

[14] CMS Collaboration, CMS-PAS-HIG-19-004, https://cds.cern.ch/record/2668684

[15] LHC Higgs Cross Section Working Group, CERN-2017-002-M (CERN, Geneva, 2016), arXiv: 1610.07922

[16] ATLAS Collaboration, ATLAS-CONF-2019-032, https://cds.cern.ch/record/2682844

[17] CMS Collaboration, Phys. Lett. B 792 (2019) 369

[18] ATLAS Collaboration, ATLAS-CONF-2019-029, https://cds.cern.ch/record/2682800

[19] G. F. Giudice, C. Grojean, A. Pomarol and R. Rattazzi, JHEP 06 (2007) 045

[20] I. Brivio and M. Trott, Phys. Rept. 793 (2019) 1

[21] B. Grzadkowski, M. Iskrzynski, M. Misiak and J. Rosiek, JHEP 10 (2010) 085

[22] ATLAS Collaboration, Phys. Rev. D 99, 072001 (2019)

[23] CMS Collaboration, Phys. Lett. B 779 (2018) 283

[24] CMS Collaboration, CMS-PAS-HIG-18-032, https://cds.cern.ch/record/2668685

[25] ATLAS Collaboration, Phys. Rev. B 784 (2018) 173

[26] CMS Collaboration, Phys. Rev. Lett. 120 (2018) 231801

[27] CMS Collaboration, CMS-PAS-HIG-18-030, https://cds.cern.ch/record/2675023

[28] CMS Collaboration, CMS-PAS-HIG-18-018, https://cds.cern.ch/record/2649208

[29] CMS Collaboration, CMS-PAS-HIG-18-019, https://cds.cern.ch/record/2649199

[30] ATLAS Collaboration, ATLAS-CONF-2019-004, https://cds.cern.ch/record/2668103

[31] ATLAS Collaboration, Phys. Rev. B 786 (2018) 59

[32] CMS Collaboration, Phys. Rev. Lett. 12 (2018) 121801 
[33] ATLAS Collaboration, JHEP 05 (2019) 141

[34] ATLAS Collaboration, CONF-HIGG-2019-028, https://cds.cern.ch/record/2682155

[35] CMS Collaboration, Phys. Rev. Lett. 122 (2019) 021801

[36] ATLAS Collaboration, CMS-PAS-HIG-18-031, https://cds.cern.ch/record/2682638

[37] ATLAS Collaboration, Phys. Rev. Lett. 120 (2018) 211802

[38] ATLAS Collaboration, arXiv:1906.02025

[39] CMS Collaboration, Phys. Rev. Lett. 122 (2019) 121803 